
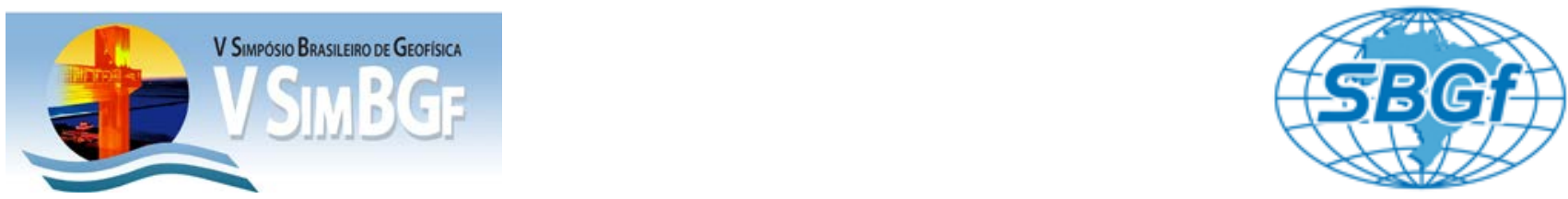

\title{
Adensamento Gravimétrico da Pista de Teste de Tietê: Estudo da Resolução, Geometria e Profundidade das Fontes
}

Lauro Augusto Ribas Teixeira - AeroGeophysica Latinoamerica - AGP-LA.

José Domingos Faraco Gallas - Instituto de Geociências - Universidade de São Saulo - USP.

Copyright 2012, SBGf - Sociedade Brasileira de Geofísica

Este texto foi preparado para a apresentação no V Simpósio Brasileiro de Geofísica, Salvador, 27 a 29 de novembro de 2012. Seu conteúdo foi revisado pelo Comitê Técnico do V SimBGf, mas não necessariamente representa a opinião da SBGf ou de seus associados. É proibida a reprodução total ou parcial deste material para propósitos comerciais sem prévia autorização da SBGf.

\section{Resume}

Airborne gravimetric systems are among geophysical systems applied to exploration. These systems rely on parametrization to gauge the quality of surveys. With the introduction of airborne gravity surveys in Brazil, with the Parnaiba Basin survey, demand for an equipment calibration lane arose.In 2004, 166 gravity stations were set in the test lane area located in the municipality of Tietê, SP. The need for more detailed geophysical models capable of identifying shallow targets resulted from surging interest in applying gravity gradiometric tensor to locate mineral deposits. The Tietê test lane was densified in order to improve the resolution limitation in tests of a range of airborne gravity systems. To achieve that, new gravity stations were set with different spacing. This stablished the first Brazilian calibration lane for scalar gravimetry and 3D airborne gravity gradiometry systems.

\section{Introdução}

Os sistemas gravimétricos aerotransportados atualmente utilizados na exploração geofísica demandam uma grande quantidade de testes para aferir a qualidade dos levantamentos executados. As principais companhias que realizam levantamentos aerogravimétricos no mundo como a AeroGeoPhysica, Carson, Bell Geospace e Fugro, executam testes de seus sistemas em laboratório e no campo, com a finalidade de parametrizar os fatores inerentes aos objetivos dos levantamentos, tais como altura de vôo, espaçamento entre linhas e a aplicação de filtros e, desta forma, garantir a qualidade e resolução dos dados adquiridos.

Com a introdução da tecnologia de sistemas gravimétricos aerotransportados na pesquisa de hidrocarbonetos no Brasil e a importância e utilidade do gradiente de gravidade na localização de objetos rasos em subsuperfície, houve a necessidade da implantação de um laboratório de campo. Este laboratório de campo, denominado de pista de teste, é onde realiza-se a calibração e aferição destes sistemas e é a área em que foram realizadas as medidas de detalhamento, objeto desta pesquisa.
Inicialmente, a pista de teste foi selecionada com base em um mapa de Anomalia Bouguer Simples do Estado de São Paulo fornecido pelo Departamento de Geofísica do IAG/USP. Foi escolhida uma área cuja variação de anomalia Bouguer é de aproximadamente $25 \mathrm{mGal}$ em uma topografia suave na região do Médio Rio Tietê. No ano de 2004 foram implementadas 166 estações gravimétricas em uma malha aproximada de $2500 \mathrm{~m} x$ 2500m na área da pista de teste.

Com a finalidade de gerar modelos geofísicos mais detalhados, melhorar o limite de resolução dos testes realizados com diferentes sistemas gravimétricos aerotransportados foi realizado um adensamento da malha gravimétrica da pista de teste.

\section{Metodologia}

Com o intuito de se tornar a primeira pista brasileira para calibração de aerogravimetria escalar e sistemas de aerogradiometria gravimétrica 3D, foram planejadas 162 novas estações gravimétricas ao longo da pista, distribuídas em diferentes espaçamentos, dispostos de acordo com o mapa de anomalias Bouguer (Figura 1) e com os dados pré-existentes obtidos no primeiro levantamento realizado em 2004 (Dal Pozzo \& Shukowsky, 2004), onde o espaçamento entre estações gravimétricas foi tomado com malha aproximada de $2500 \mathrm{~m} \times 2500 \mathrm{~m}$, que corresponde a um levantamento na escala 1:500.000 (Sazhina \& Grushinsky, 1971 apud Vasconcellos et al., 1994).

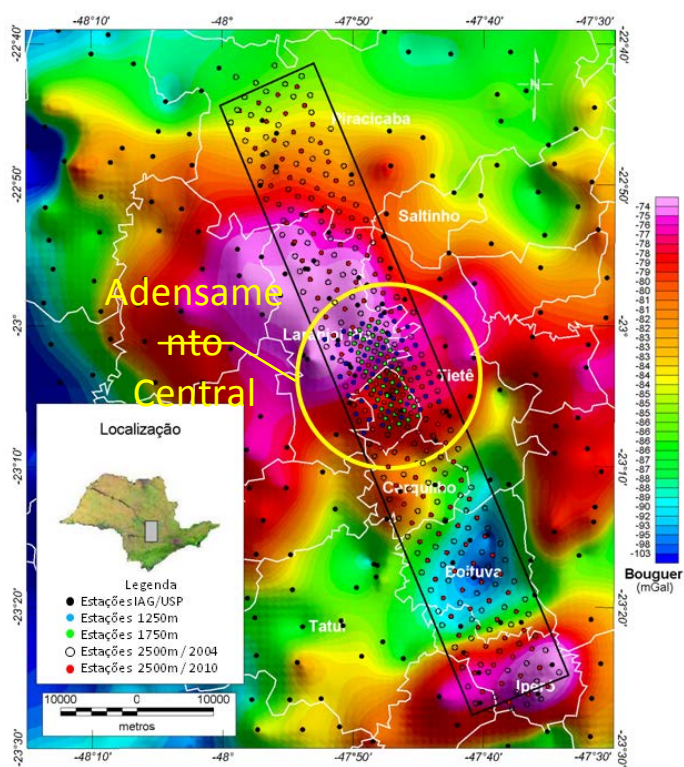

Figura 1 - Mapa de Anomalia Bouguer. 
A execução deste levantamento foi acompanhada de rigoroso levantamento planialtimétrico de forma a permitir a precisão necessária para as correções inerentes ao processamento dos dados. Este levantamento foi efetuado utilizando-se um GPS diferencial e na execução do trabalho foram utilizados os métodos de posicionamento Relativo Estático e Relativo Estático Rápido.

O posicionamento relativo estático foi aplicado na determinação das estações fixas, estabelecidas nas cidades de Boituva e Conchas, amarradas ao RBMC Rede Brasileira de Monitoramento Contínuo do IBGE, pertencente ao Sistema Geodésico Brasileiro (SGB). O posicionamento relativo estático-rápido foi utilizado no adensamento em bases com distâncias entre os receptores iguais ou inferiores a $20 \mathrm{~km}$. Os pontos gravimétricos foram determinados utilizando 0 equipamento CG-5 da Scintrex, adensando os pontos a partir do levantamento gravimétrico executado em 2004, executado com 0 equipamento Lacoste\&Romberg modelo $G$. A referência gravimétrica foi calculada a partir da Rede Gravimétrica do Sistema Geodésico Brasileiro estabelecido pelo IBGE - Instituto Brasileiro de Geografia e Estatística. Desta forma, no adensamento atual, foi executado um levantamento gravimétrico, envolvendo uma área aproximada de $500 \mathrm{~km}^{2}$ com diferentes espaçamentos que variaram entre $2500 \mathrm{~m}, 1750 \mathrm{~m}$ e 1250m (Figura 1).

\section{Resultados}

Com o resultado obtido após a compilação e processamento dos dados, empregando-se um sistema GPS diferencial e a aplicação das reduções gravimétricas, assim como os procedimentos de ajuste polinomial executados com aplicação de filtros 2D utilizando a FFT (Fast Fourier Transform) foram gerados os grids do campo residual e regional, no programa OASIS MONTAJ 5.1 da GEOSOFT, com o objetivo de separar os efeitos de anomalias de gravidade produzidos por feições profundas e feições rasas.

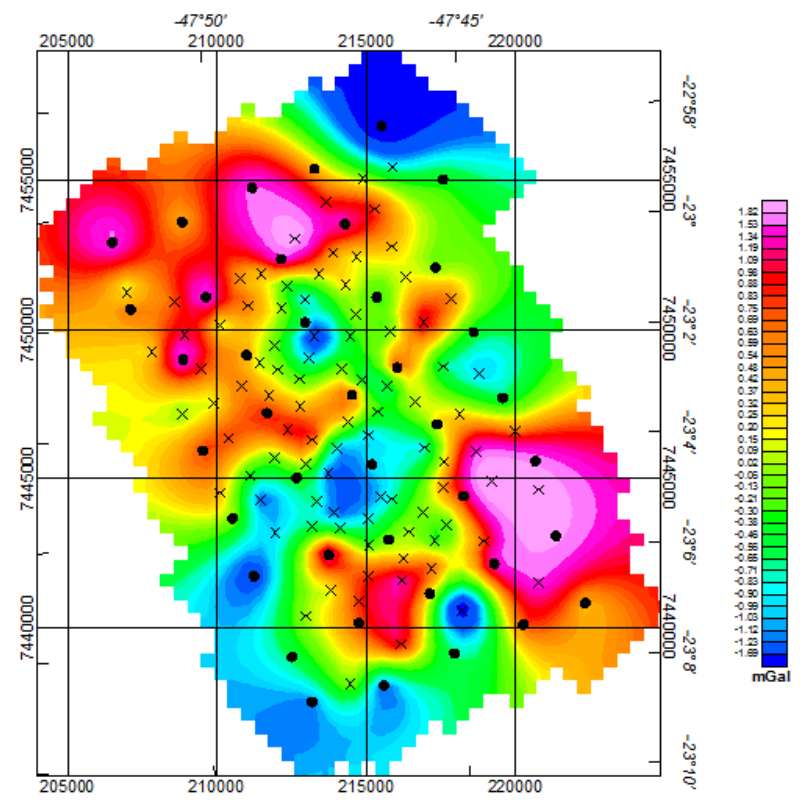

Figura 2 - Campo Residual do adensamento central.
Fontes de origem mais profundas, como os relevos do embasamento de uma bacia sedimentar, apresentam um grande comprimento de onda, caracterizando estruturas regionais. Uma anomalia regional ampla, de variação suave, normalmente sobrepõe-se às anomalias locais, de comprimento de onda mais curto produzidos por feições mais rasas. Com o objetivo de melhorar a delineação de feições rasas, foi realizada uma análise mais detalhada na região central da pista a partir de dados existentes, que apresentaram maior variação do gradiente gravimétrico, dados levantados em campo, e dados geológicos já disponíveis. Recentes desenvolvimentos nos sistemas de aerogradiometria têm resultado em interesse renovado nas medidas do gradiente da gravidade, especialmente na delineação de corpos anômalos rasos, como, por exemplo, corpos mineralizados (Jekeli, 1988; Vasco, 1989). Dado o interesse no estudo das anomalias locais na área do adensamento central, a remoção do campo regional é fundamental para a interpretação e modelagem dos dados. Existem vários métodos para remoção do campo regional, dentre eles pode-se citar os métodos gráficos, espectrais e análise da superfície de tendência. A remoção do regional utilizando a análise da superfície de tendência através do ajuste polinomial é uma das técnicas mais utilizadas na determinação do campo residual (Figura 2). Nesse método a superfície é expressa matematicamente por um polinômio bidimensional com seu grau variando de acordo com o campo regional a ser ajustado. Através da comparação das médias radiais dos espectros de potência foi realizada a análise da contribuição do adensamento na estimativa de profundidade das fontes na área da pista de teste. Todo o procedimento de cálculo foi realizado no programa OASIS MONTAJ 5.1 da GEOSOFT. A transformada rápida de Fourier (FFT) foi a metodologia utilizada para converter os dados no domínio do espaço para o domínio da frequência. As profundidades das fontes foram estimadas a partir da análise do espectro de potência, pelo método de Spector \& Grant (1970). A Figura 3 mostra a diferença entre as profundidades obtidas com os dados pré-existentes de 2004 e as profundidades encontradas com 0 adensamento realizado em 2010. Tanto para a área de adensamento $2500 m$ e central, foi possível estimar a profundidade de fontes mais rasas devido a maior resolução dos dados. $\mathrm{Na}$ área do adensamento $2500 \mathrm{~m}$, as fontes profundas e médias foram mais bem amostradas, mas continuam praticamente no mesmo intervalo, por volta de $6 \mathrm{~km}$ e 1,8 $\mathrm{km}$, respectivamente. Já as fontes rasas, foram posicionadas nos níveis de altos do embasamento cristalino e possíveis intrusivas da Formação Serra Geral, porém com resolução insuficiente para delimitar a geometria dos corpos. Na área do adensamento central, as fontes rasas foram estimadas nos níveis das rochas intrusivas (cerca de $300 \mathrm{~m}$, contra $500 \mathrm{~m}$ dos dados de 2004), mostrando que a resolução atual dos dados é suficiente para a avaliação de dados aerogradiométricos.

\section{Discussão e Conclusões}

Para avaliar se a resolução espacial dos dados do adensamento central é compatível do ponto de vista do 
conteúdo espectral com as rochas intrusivas da Formação Serra Geral, principal fonte gravimétrica intrassedimentar, a título de exemplo foi executada uma modelagem 3D (Tomografia Gravimétrica), com o método inverso, utilizando o programa DVOP, do pacote SIGMA 3D.

O sill do bairro Taquaral, localizado no Km 163 da Rodovia Marechal Rondon foi utilizado como exemplo de estrutura geológica que foi adequadamente amostrada pelo adensamento central. Os valores de contraste de densidade, nos intervalos médios, são condizentes com as densidades das rochas intrusivas, diabásio, no caso, que têm densidades médias entre 2,8 a $3,0 \mathrm{~g} / \mathrm{cm}^{3}$, e os sedimentos do Subgrupo Itararé, com densidades médias entre 2,3 a 2,5 g/ $\mathrm{cm}^{3}$. A Figura 4 apresenta o modelo 3D obtido a partir dos dados do campo residual, com a distribuição geométrica dos contrastes de densidade para as profundidades entre 0 e 700 metros, em perfis rebatidos equidistantes de $1000 \mathrm{~m}$. A partir dos resultados obtidos com o pós-processamento dos dados empregando-se um sistema GPS diferencial e a aplicação das reduções gravimétricas, foram obtidos mapas das anomalias gravimétricas, bem como as interpretações a respeito dos mesmos. O adensamento da malha gravimétrica da pista de testes de Tietê contribuiu na geração de modelos geofísicos mais detalhados melhorando o limite de resolução na parametrização de diferentes sistemas gravimétricos aerotransportados.

\begin{tabular}{|c|c|c|c|}
\hline \multicolumn{4}{|c|}{ ESTIMATIVA DE PROFUNDIDADE - ADENSAMENTO 2500m } \\
\hline FONTES & 2004 & 2010 & DIFERENÇA \\
\hline Rasas & $0,50 \mathrm{~km}$ & $0,33 \mathrm{~km}$ & $\mathbf{0 , 1 7} \mathrm{km}$ \\
\hline Médias & $2,00 \mathrm{~km}$ & $1,43 \mathrm{~km}$ & $\mathbf{0 , 5 7} \mathrm{km}$ \\
\hline Profundas & $\mathbf{6 , 2 4} \mathrm{km}$ & $\mathbf{5 , 8 9} \mathrm{km}$ & $\mathbf{0 , 3 5} \mathrm{km}$ \\
\hline \multicolumn{4}{|l}{} \\
\hline ESTIMATIVA DE PROFUNDIDADE - ADENSAMENTO CENTRAL \\
\hline FONTES & 2004 & 2010 & DIFERENÇA \\
\hline Rasas & $\mathbf{0 , 5 5} \mathrm{km}$ & $\mathbf{0 , 3 7} \mathrm{km}$ & $\mathbf{0 , 1 3} \mathrm{km}$ \\
\hline Médias & $\mathbf{1 , 6 0} \mathrm{km}$ & $\mathbf{1 , 2 1} \mathrm{km}$ & $\mathbf{0 , 1 1} \mathrm{km}$ \\
\hline
\end{tabular}

Figura 3 - Estimativa de Profundidades.

No adensamento realizado em toda extensão da pista, comparando-se com os dados pré-existentes de 2004, observa-se que a resolução para as fontes médias e profundas foi pouco alterada, mas houve uma melhora na resolução das fontes rasas.

$\mathrm{Na}$ área do adensamento central, região com maior densidade de pontos, a resolução foi mais significativa, sendo possível delimitar as profundidades e geometria das fontes rasas. Como exemplo, as rochas intrusivas anteriormente estimadas em cerca de $500 \mathrm{~m}$, evoluíram para uma precisão da ordem de $300 \mathrm{~m}$. A partir do esboço geofísico-geológico apresentado com base no adensamento central, conclui-se que a resolução atual dos dados é adequada à avaliação de dados de gradiometria gravimétrica aérea, amplamente utilizada em prospecção mineral para elaboração de modelos geológicos.

Desta forma, foi conferida à pista de testes a aplicabilidade para essa metodologia de investigação geofísica, satisfazendo os objetivos deste projeto de pesquisa.

\section{Agradecimentos}

Aos professores e ao programa de Pós-Gradução em Recursos Minerais e Hidrologia e ao Departamento de Geologia Sedimentar e Ambiental da IGc-USP pelas importantes recomendações e apoio técnico e a todos colegas da AGP-LA, empresa responsável pelo crescimento profissional do autor Lauro Ribas.

\section{Referências}

Dal Pozzo, H.A.P, Shukowsky,W, 2004. Construção e Desenvolvimento da Pista de Testes de Aerogravimetria de Tietê - Projeto Levantamentos Aerogeofísicos da Bacia do Parnaíba ANP- USP. Internal publication, 55p.

Instituto Brasileiro de Geografia e Estatística, 2005. RBMC - Rede Brasileira de Monitoramento Contínuo. Rio de Janeiro (RJ). Disponível em:

$<$ http://www.ibge.gov.br/home/geociencias/geodesia/rbmc /rbmc.shtm.>Acesso em: 20 de ago. 2010.

Jekeli C. 1988. The Gravity Gradiometer Survey System (GGSS). EOS Transaction of the American Geophysical Union,69: 116-117.

Sazhina, N., and Grushinsky, N., 1971. Gravity Prospecting. Change of Gravity with Time. Tides., MIR Publishers, Moskow, p. 109 - 121.

Spector, A. and F.S. Grant, 1970. Statistical models for interpreting aeromagnetic data, Geophysics, 35, 293-302.

Vasco DW. 1989. Resolution and variance operators of gravity and gravity gradiometry. Geophysics, 54: 889899.

Vasconcelos, R. M.; Metelo, M. J.; Motta, A. C.; Gomes, R. D. - 1994. Geofísica em Levantamentos Geológicos no Brasil.CPRM, Rio de Janeiro. 


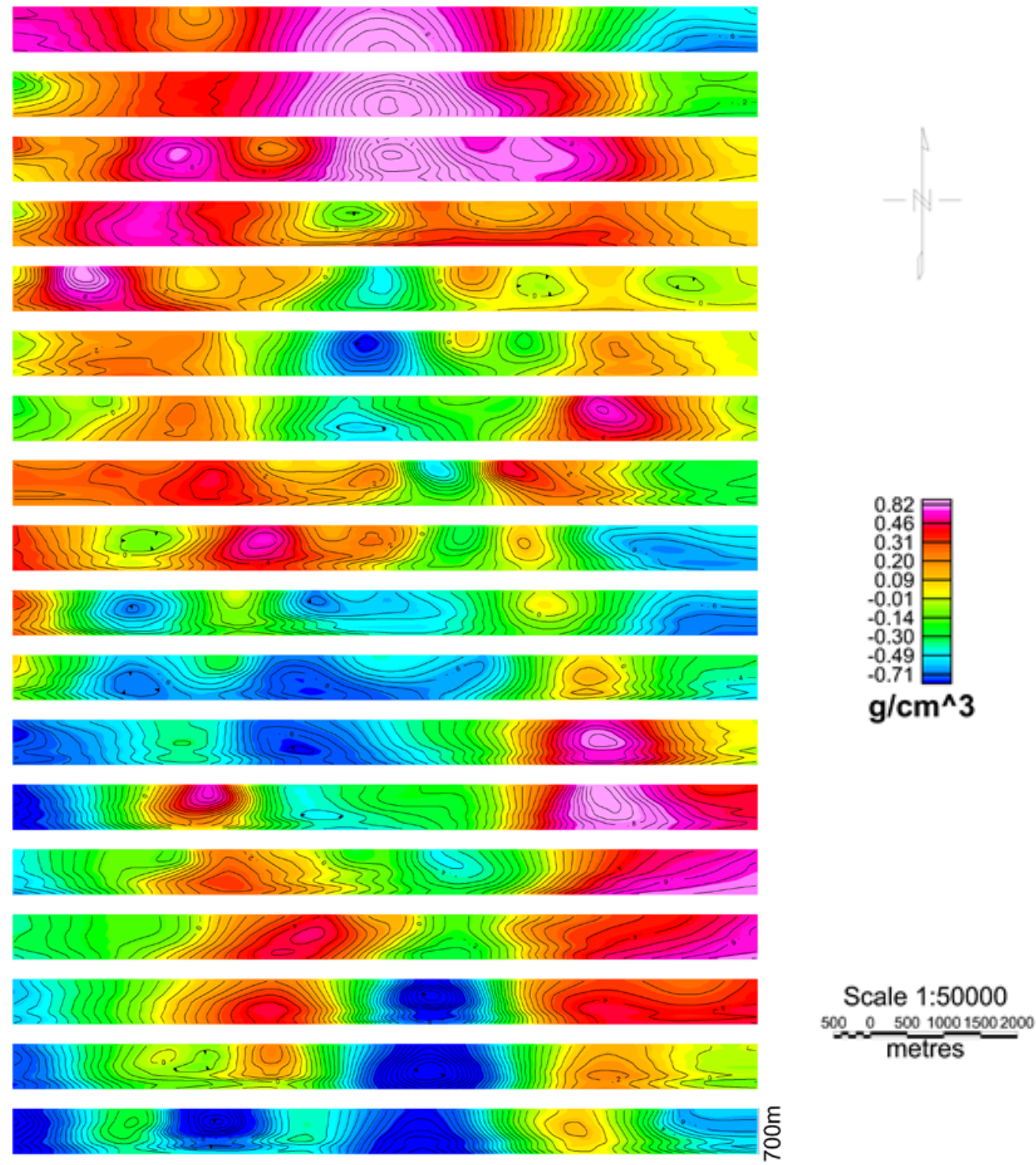

Figura 4 - Modelagem 3D. 